\title{
$\operatorname{Conf}-950232.21$
}

Environmental Restoration and Waste Management Program

\section{SIMULATION ANALYSIS OF CONTROL STRATEGIES FOR A TANK WASTE RETRIEVAL MANIPULATOR SYSTEM*}

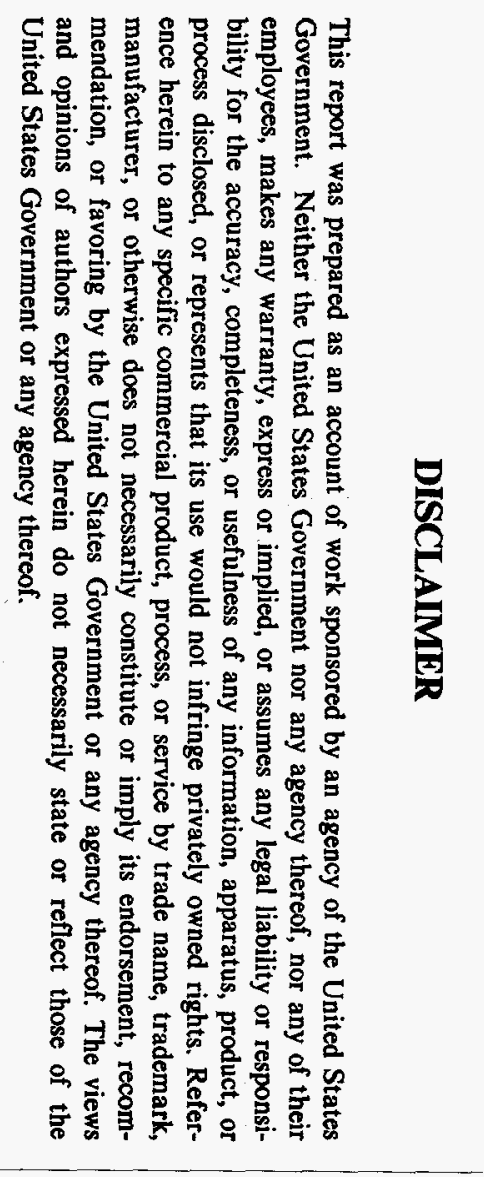

\author{
J. C. Shryver \\ Engineering Physics and Mathematics Division \\ Oak Ridge National Laboratory $\dagger$ \\ Post Office Box 2008 \\ Oak Ridge, Tennessee 37831-6360 \\ J. V. Draper \\ Robotics and Process Systems Division \\ Oak Ridge National Laboratory $\dagger$ \\ Post Office Box 2008 \\ Oak Ridge, Tennessee 37831-6304
}

\begin{abstract}
The submitted manuscript has been authored by a contractor of the U.S

authored by a contractor of the U.S.

$840 R 21400$. Accordingly, the U.S.

$84 O R 21400$. Accordingly, the U.S.
Government retains a paid-up, Government retains a paid-up,
nonexclusive, irrevocable, worldwide license to publish or reproduce the published form of this contribution, prepare derivative works, distribute copies

to the public, and perform publicly and

dispiay publicly, or allow others to do so,

for U.S. Government purposes.
\end{abstract}

To be presented at the American Nuclear Society Sixth Topical Meeting on Robotics and Remote Systems Monterey, California February 5-10, 1995

*Research sponsored by the Office of Environmental Restoration and Waste Management, U.S. Department of Energy, under contract DE-AC05-84OR21400 with Martin Marietta Energy Systems, Inc.

† Managed by Martin Marietta Energy Systems, Inc., under contract DE-AC05-84OR21400 with the U.S. Department of Energy.

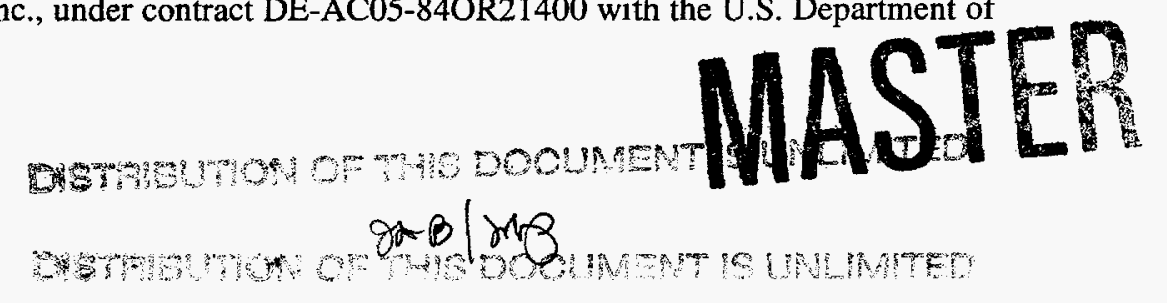




\section{DISCLAIMER}

Portions of this document may be illegible in electronic image products. Images are produced from the best available original document. 


\section{SIMULATION ANALYSIS OF CONTROL STRATEGIES FOR A TANK WASTE RETRIEVAL MANIPULATOR SYSTEM}

\author{
J. C. Schryver \\ Engineering Physics and Mathematics Division \\ Oak Ridge National Laboratory \\ Post Office Box 2008 Mail Stop 6360 \\ Oak Ridge, Tennessee 37831-6360 \\ Voice: (615) 574-4710 \\ schryverjc@ornl.gov
}

\begin{abstract}
A network simulation model was developed for the Tank Waste Retrieval Manipulator System, incorporating two distinct levels of control: teleoperation and supervisory control. The model included six error modes, an attentional resource model, and a battery of timing variables. A survey questionnaire administered to subject matter experts provided data for estimating timing distributions for level of control-critical tasks. Simulation studies were performed to evaluate system behavior as a function of control level and error modes. The resuits provide important insights for development of waste retrieval manipulators.
\end{abstract}

\section{INTRODUCTION}

This paper describes the methodology and results of a network simulation study of the Tank Waste Retrieval Manipulator System (TWRMS) to be employed for underground storage tank remediation. The development of the simulation model was based upon the results of a task analysis for the same system and preserves the network structure developed during the previous study' and presented in detail elsewhere. ${ }^{2}$ This study was performed by the Oak Ridge National Laboratory (ORNL), in the Robotics and Process Systems Division and Engineering Physics and Mathematics Division. Funding was provided the U. S. Department of Energy's Office of Technology Development, Robotics Technology Development Program.

Simulation can be used to support a quantitative performance evaluation of proposed control systems for the TWRMS, even before these systems are installed at the site. The two most frequently mentioned control systems

\author{
J. V. Draper \\ Robotics and Process Systems Division \\ Oak Ridge National Laboratory \\ Post Office Box 2008 Mail Stop 6304 \\ Oak Ridge, Tennessee 37831-6304 \\ Voice: (615) 574-5478; Facsimile: (615) 574-5479 \\ draperjv@ornl.gov
}

are teleoperation and supervisory control. Teleoperation requires continuous manual positioning of the manipulator and end-effector for waste retrieval operations. Supervisory control requires the user to program and verify control maneuvers off-line before allowing the automatic control system to execute the maneuvers. In both cases a human user monitors system performance with the assistance of video monitors, although in supervisory control the human user may instead monitor a graphic world model of the tank interior and manipulator.

The simulation assumed best case scenarios for both control modes. For the case of teleoperation, it was assumed that the equipment was reliable and did not malfunction. In addition, under supervisory control, it was assumed that graphic world models provided sufficient detail for reliable programming and that effective interactive programming techniques were available to assist the user. For all levels of control, sensor capabilities were assumed to be adequate in a hostile tank environment (i.e., coverage from multiple perspectives was sufficient and visibility was acceptable during all phases of waste processing).

\section{METHOD}

The TWRMS simulation model was developed with the MicroSAINT package, a network simulation modeling tool kit for personal computers. Network simulation is a suitable tool for modeling systems that can be decomposed into a set of discrete chronological steps or tasks. A set of tasks and pathways (which connect tasks according to their precedence relationships) constitute the network. There are important advantages of decomposing a complex system into smaller steps: it is often easier to describe the 
behavior of constituent parts of a process than to describe the whole, and the performance of the whole system can be studied by varying the behavior of the constituent parts. The set of steps may be organized as an abstraction hierarchy, where the top level reveals the most general view of the operation of the network. Each box in the top level contains a subnetwork, which in turn may contain its own subnetwork, etc. Successive levels in the hierarchy show more detail of smaller parts of the entire system.

A network diagram shows the constituent parts of the simulation model. It is essentially a task block flow diagram where nodes represent activities of the system. In order to create a network, a task analysis is performed to identify the activities of the system. The network also shows the path or paths followed after completion of each activity. The network diagram can be implemented as a computer-based network simulation model using a network simulation package such as MicroSAINT.

Simple networks process tasks one at a time in sequential fashion. More complex networks allow multiple entities to propagate through the network in parallel fashion. Thus it is possible for multiple tasks to be processed concurrently in such networks. Tactical and multiple branching also create the possibility that entities will follow novel paths through the network on different simulation runs. Ref. 3 lists examples network modeling for complex manned systems and Ref. 4 for a simple example of a MicroSAINT model application in nuclear power plant control.

\section{Simulation Model Development for the TWRMS}

A simulation model was developed to assess the operation of the TWRMS for underground storage tank remediation. Constructing a network model began with problem definition and identification of primary questions to be answered. The main issues for the TWRMS were:

1. Does level of control affect system performance? Specifically, does either teleoperation or supervisory control produce superior performance characteristics?

2. How does the likelihood of various system and user errors affect performance curves under teleoperation and supervisory control?

3. Are the effects of level of control and error rates independent of the characteristics of the tank?

The first issue can be addressed independently for sub-tasks like riser cutting and removal of cut-up risers, as well as for total mission duration. The second issue is important as it may qualify the conclusions developed for level of control. Requirements or upper limits may also be established for specific error rates through an analysis of performance decrements. The establishment of error rate limits may lead to further requirements for system reliability and user training. The third issue bears on the generalizability of findings generated by the simulation model.

Task Network. The simulation model is based upon the task analysis described in Ref. 1. At root level, the model captures insertion of the TWRMS equipment into the tank, followed by removal of waste layers, and concludes with the removal of the TWRMS equipment from the tank (these may be termed mission phases). The major functions of the first mission phase are:

1. Activate auxiliary closed-circuit television (CCTV) system

2. Insert auxiliary CCTV system into tank

3. Inspect tank interior

4. Develop riser cutting strategy

5. Activate TWRMS system

6. Insert TWRMS

The major functions of the waste removal phase are:

1. Cut risers/In-Tank Hardware (ITH)

2. Remove cut-up risers/TTH

3. Characterize waste

4. Remove waste layer

5. Remove residual waste

The final mission phase has two main functions:

1. Remove TWRMS

2. Remove auxiliary CCTV system

Error Modeling. In order to study the robustness of different levels of control of the TWRMS, perturbations in system performance are evaluated through introduction of various error modes into the task network. Six errors were identified for the second mission phase: (1) small robotic position error, (2) large robotic position error, (3) user position error, (4) grasping error, (5) release error, and (6) monitoring error. The first two errors were associated with robotic maneuvers, and the last four errors are user errors. Space constraints will limit the present discussion to the first five errors.

Position errors are associated with manipulator movement and end-effector operation in the vicinity of a 
riser/TTH that is about to be cut or retrieved for placement in the hopper. These errors can also occur while preparing to initiate waste removal operations. Small robotic (and user) position errors are assumed to be correctable by manual intervention. A new attempt to complete the operation is initiated after repositioning. Large robotic position errors are routed back to teaching mode for correction. Reteaching may be necessary, for example, if the program selected the wrong riser as the next item in a cutting or removal sequence. User position errors are possible in both teleoperation and supervisory control modes if manual intervention is used to reposition the manipulator.

Position errors may also precede damage to the manipulator or end-effector. Damage is most likely to occur during transit when the manipulator collides with some object or riser. Even if the manipulator does not sustain substantial damage, the system must be halted and inspected to determine the extent of damage. If the damage is extensive, the manipulator is withdrawn from the tank and the equipment is repaired or the end-effector is replaced.

Grasping error occurs when the end-effector, under teleoperation, is prompted to grasp an item while out of position. The result is that the item grasped is not under firm control, and the item is dropped. The manipulator is then repositioned to regrasp the item.

Release error refers to premature release of a cut-up riser piece. It is assumed that the piece drops to the waste surface instead of into the hopper. There is also the chance that damage to the manipulator will be sustained during an out-of-position release. Damage is inspected, and if it is extensive, the manipulator is withdrawn from the tank for repair or end-effector replacement. The dropped item is then recovered.

Independent Variables. The two major independent variables were level of control and tank type. Two levels of control were instantiated into the simulation model: supervisory control and teleoperation (manual) control. Two tanks were modeled for the TWRMS simulation, based on information about existing tanks at the USDOE Hanford site. Table 1 presents the tank parameters.

Table 1. Tank parameters in the model.

\begin{tabular}{l|ll}
\hline Parameter & Tank I & Tank 2 \\
\hline Diameter & $22.86 \mathrm{~m}$ & $22.86 \mathrm{~m}$ \\
Height & $5.49 \mathrm{~m}$ & $7.32 \mathrm{~m}$ \\
Waste Depth & $30.48 \mathrm{~cm}$ & $121.92 \mathrm{~cm}$ \\
No. of Risers & 4 & 23 \\
\hline
\end{tabular}

Dependent Measures. The primary criterion for evaluation of system performance was the duration of the mission. The times required to complete several subfunctions were also of interest. The model tracked the total time to perform the following activities:

1. Insert the CCTV

2. Insert the TWRMS

3. Develop cut strategy

4. Inspect the tank

5. Cut risers/ITH

6. Remove cut-up risers/TTH

7. Characterize waste

8. Remove waste

9. Remove TWRMS

10. Remove CCTV

\section{Input Parameter Data}

Since there are literally hundreds of tasks in the TWRMS network, it was important to adopt a set of reasonable equivalence assumptions for task timing. The following list of assumptions were made in the model:

1. Insert CCTV and Remove CCTV are reversible functions with equal time distributions.

2. Insert TWRMS and Remove TWRMS are reversible functions with equal time distributions.

3. Teach Cut and Teach Removal have equal time distributions.

4. Adjust Position in Manual Cut and Manual Removal have equal time distributions.

5. Adjust Position in Robotic Cut and Robotic Removal have equal time distributions.

6. Robotic and manual cutting operations have identical time distributions.

7. The time to grasp an item is identically distributed for supervisory control and teleoperation.

8. Waste removal rate was a uniform 113.55 liters/minute with no regard to waste form (waste in the real tanks may be sludge, damp salt cake, dried salt cake, or wet salt cake, or any combination).

Other task timing input data were developed through best engineering judgment or task timing survey data provided by subject matter experts. Most task times were modeled by gamma distributions, which require two parameters to define their exact shape. The survey was administered to 5 subject matter experts. Three were ORNL staff and two were affiliated with Pacific Northwest Laboratories. Survey questions were limited to tasks that were critical for evaluation of level of control. The final timing parameters are reproduced in Table 2. 


\section{Experimental Design for Parametric Study}

Seven parametric simulation studies were designed to evaluate system behavior as a function of control level and various error modes; four of them are reported here. Default values were used for model variables wherever they were not systematically varied in a particular design. Each condition was run 20 times under a different random seed for each block design.

Level of Control X Tank Type (2X2). The two levels of control were teleoperation and supervisory control. Both tank types were tested in this study.

Robotic Position Error Probability (5X1). These runs occurred under supervisory control using Tank 1 only. The probability of large and small position errors were covaried in the design such that $e=\{0.005,0.05$, $0.075,0.125,0.25\}$ for both small and large probabilities.

User Position Error Probability (5X1). These runs occurred under teleoperation control using Tank 1 only. The probability of user position error was systematically varied over five sets of runs such that $e=\{0.01,0.10$, $0.15,0.25,0.50\}$.

Damage Probability X Level of Control (5X2). These runs used Tank 1 only. The two levels of control were teleoperation and supervisory control. The probability of damage resulting to the manipulator or end-effector

Table 2. Gamma parameters for crititcal tasks (min).

\begin{tabular}{|c|c|c|}
\hline Task & Mean & Std. Dev. \\
\hline $\begin{array}{l}\text { Adjust position on graphical world } \\
\text { model }\end{array}$ & 0.28 & 0.20 \\
\hline $\begin{array}{l}\text { Monitor replay of a single reposi- } \\
\text { tion/cut }\end{array}$ & 1.46 & 0.75 \\
\hline Perform a 6-inch riser cut & 0.78 & 0.45 \\
\hline $\begin{array}{l}\text { Manually position manipulator for } \\
\text { cut/retrieval }\end{array}$ & 1.10 & 0.70 \\
\hline $\begin{array}{l}\text { Robotically position manipulator for } \\
\text { cut/retrieval }\end{array}$ & 1.54 & 0.75 \\
\hline $\begin{array}{l}\text { Manually reposition manipulator after } \\
\text { miscue }\end{array}$ & 1.13 & 0.68 \\
\hline $\begin{array}{l}\text { Readjust position on graphical world } \\
\text { model after error }\end{array}$ & 0.42 & 0.27 \\
\hline $\begin{array}{l}\text { Repair manipulator or exchange end- } \\
\text { effector }\end{array}$ & 513.00 & 285.00 \\
\hline Grasp a cut-up riser piece & 1.45 & 0.84 \\
\hline $\begin{array}{l}\text { Manually transfer a riser piece to the } \\
\text { hopper }\end{array}$ & 1.76 & 0.97 \\
\hline $\begin{array}{l}\text { Robotically transfer a riser piece to the } \\
\text { hopper }\end{array}$ & 0.72 & 0.40 \\
\hline
\end{tabular}

after a large position error was systematically varied over the set of values $e=\{0.001,0.005,0.01,0.05,0.10\}$ for both control modes.

\section{RESULTS}

Analysis of Variance (ANOVA) and linear regression tests were performed to determine the statistical significance of independent variables tested in parametric studies of the TWRMS simulation model. ${ }^{5}$ A statistically significant result implies that we can be reasonably confident that differences observed among group means actually exist in the population (of the simulated world), and are not simply statistical aberrations generated by chance alone. Probability levels associated with calculated F-ratios are expressed in parentheses to indicate the actual probability that observed differences among means could have occurred by chance.

\section{Mission Duration}

Figure 1 shows mission duration for each level of control. Teleoperation resulted in a mean mission duration of 2.47 shifts, compared to 2.59 shifts for supervisory control for Tank 1. However, a clear and significant distinction was found between teleoperation (10.55 shifts) and supervisory control (13.02 shifts) control in Tank 2 ( $p$ $<0.0001$ ). Supervisory control resulted in substantially longer missions than teleoperation, but the relative timing advantage of teleoperation was more evident in the simulation runs conducted using the larger tank.

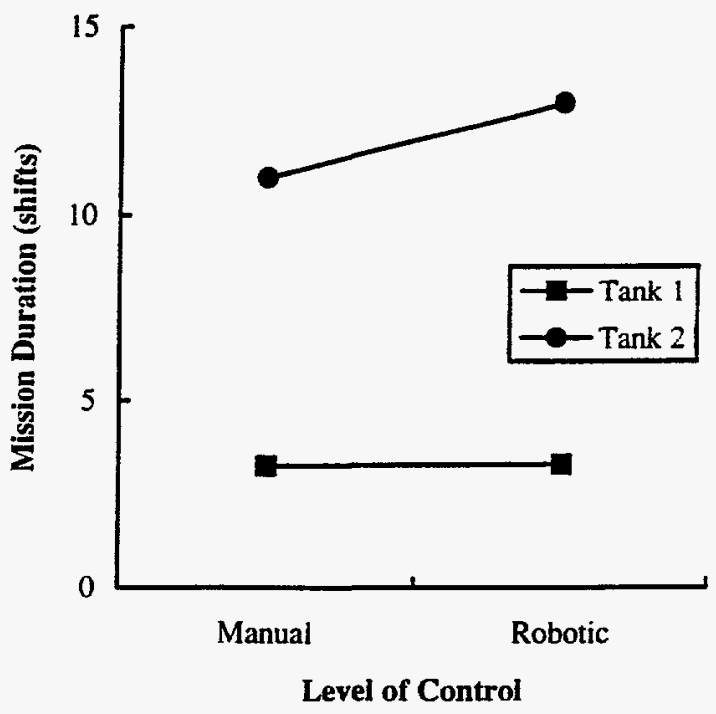

Figure 1. Interaction of control level and tank type on shifts. 
Riser cutting time $(p<0.0001)$ and piece retrieval time ( $p<0.035$ ) were both significantly longer under supervisory control as compared to teleoperation. Mean cutting time was 213 (teleoperation) and 283 minutes (supervisory control) for Tank 1, and 1584 (teleoperation) and 2664 minutes (supervisory control) for Tank 2. Mean piece retrieval time was 170 (teleoperation) and 228 minutes (supervisory control) for Tank 1, and 1993 (teleoperation) and 2199 minutes (supervisory control) for Tank 2.

Operator attentional effort was modeled as a weighted linear combination of the cumulative times allocated to different classes of activities, which were scaled using a subjective magnitude estimation procedure. Normalized values for attentional effort ranged between 0 (low) and 100 (high). The ANOVA showed a significant interaction between level of control and tank type. For Tank 1, mean attentional effort was greater under manual control (48.6) as compared to supervisory control (41.8). This trend was more pronounced for Tank 2, where mean attentional effort was respectively 50.7 and 38.9 for manual and supervisory control. The addition of waste layers apparently eased attentional demand under supervisory control, but increased the effort required for manual operations.

\section{Robotic Position Error Probability (RPEP)}

Mission duration increased significantly $(p<0.0001)$ as a function of RPEP. A Student-Newman-Keuls posthoc test (which considers all pairwise comparisons among group means) revealed that $\mathrm{RPEP}=0.5$ generated a mean mission duration significantly different from all other RPEP levels. Figure 2 shows that, except for the small jump in mission duration from RPEP $=0.10$ to 0.15 , the curve is relatively flat until RPEP reaches the rather extreme value of 0.5 . Thus, for a moderate range of values, the effect of RPEP on mission duration is quite modest.

Riser cutting time and retrieval time increased significantly ( $p<0.0001$ ) with RPEP as shown in Figure 3. Retrieval time had a much steeper slope, indicating that it was more sensitive to RPEP than cutting time. However, for values of RPEP under 0.25 , its effect on retrieval time was not substantial. Values of RPEP under 0.5 did not have a significant impact on cutting time. Waste removal time was not affected by RPEP.

\section{User Position Error Probability (UPEP)}

Mission duration increased significantly $(p<0.0001)$ as a function of UPEP. Group means are plotted in Figure 4. As with RPEP, a Student-Newman-Keuls post-hoc test

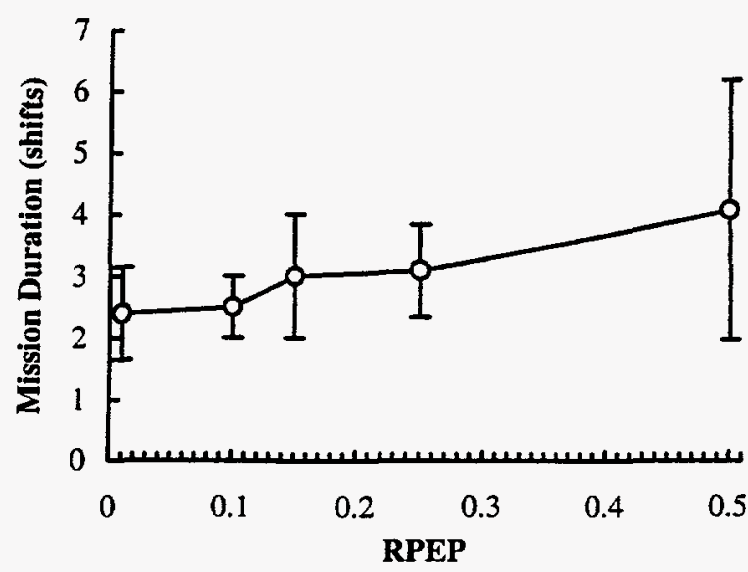

Figure 2. Impact of RPEP on mission duration.

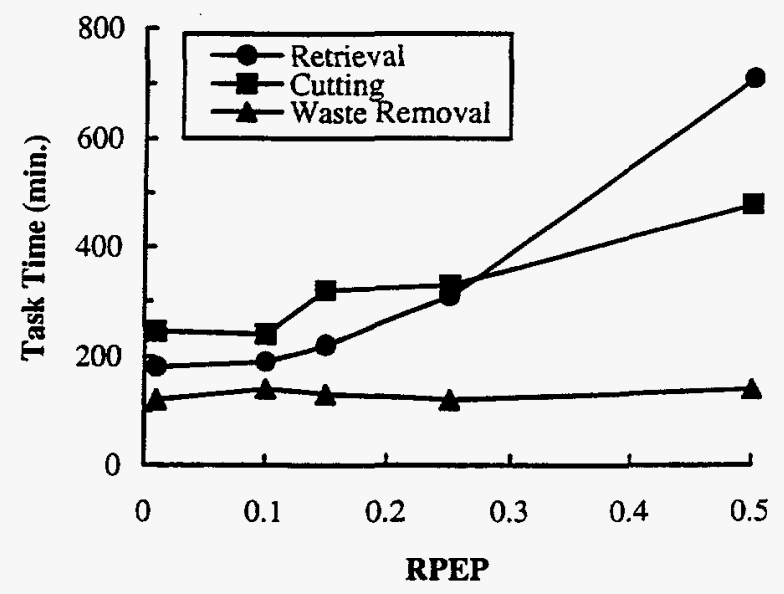

Figure 3. Impact of RPEP on task time.

revealed that UPEP $=0.5$ generated a mean mission duration significantly greater than the other UPEP levels. It appeared that for a moderate range of UPEP up to 0.25 , the effect on mission duration was not of practical significance.

Riser cutting time and retrieval time increased significantly ( $p<0.0001$ ) with UPEP as shown in Figure 5. The curves for retrieval time and cutting time had similar slopes, and were affected in much the same manner by UPEP. Again, the effects of UPEP on cutting time and retrieval time were most evident for UPEP $=0.5$. Waste removal time was not affected by UPEP. Total repair time increased significantly with UPEP, as expected $(\mathrm{p}=0.0071)$. 


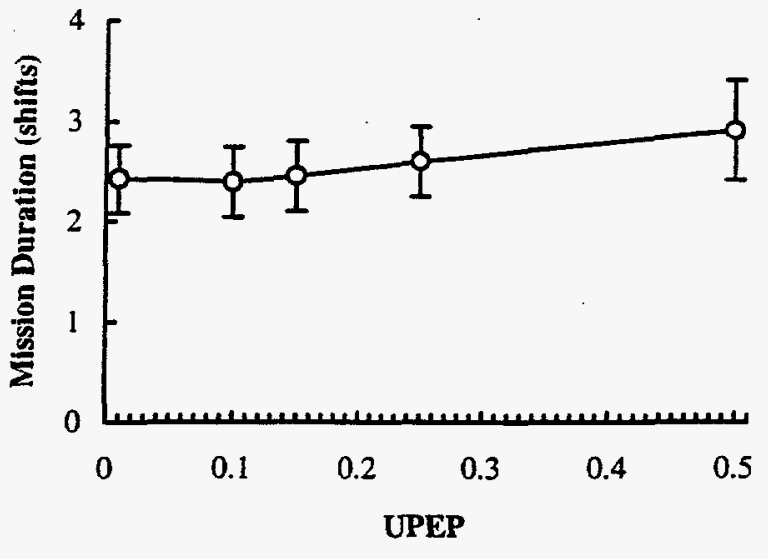

Figure 4. Impact of UPEP on mission duration.

\section{Damage Probability (DMGPROB)}

From Figure 6, mean mission duration increased by about $50 \%$ in the interval from DMGPROB $=0.001$ to 0.1 . At high levels of DMGPROB ( 0.1$)$ the differences between teleoperation and supervisory control were obscured.

Cutting time, piece retrieval time, and waste removal time increased significantly as a function of DMGPROB ( $p$ $<0.0012$ ). In general, the main effect of DMGPROB held for both supervisory control (Figure 7) and teleoperation (Figure 8). The single exception was cutting time under teleoperation, which was relatively unaffected by DMGPROB.

\section{SUMMARY AND CONCLUSIONS}

The findings for level of control demonstrate a clear advantage of teleoperation as compared to supervisory control of the TWRMS for both riser cutting and piece retrieval operations, as well as for the mission as a whole. This result was replicated for both the small and large tank, although differences were more apparent in waste processing conducted in the large tank. The timing differences between teleoperation and supervisory control for the small tank were not significant.

Comparison of timing distributions demonstrates the underlying causes of the findings for level of control. Mean times required to transfer a cut-up riser piece to the hopper were $1.76 \mathrm{~min}$ and $0.72 \mathrm{~min}$ respectively for teleoperation and supervisory control. Transfer to the hopper was faster under supervisory control. Teleoperation was also subject to user errors in grasping and releasing riser pieces into the hopper, whereas supervisory control did not explicitly model these errors. These considerations tended to yield the advantage to supervisory control.

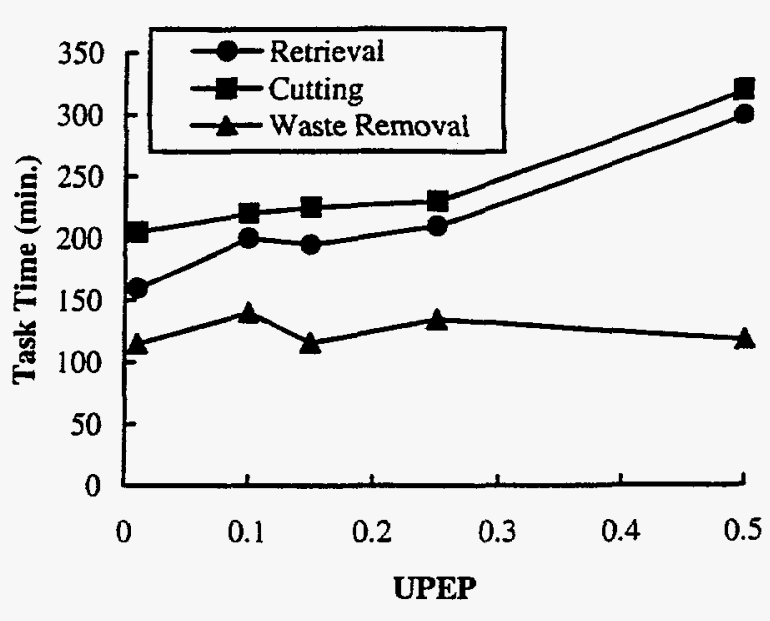

Figure 5. Impact of UPEP on task time.

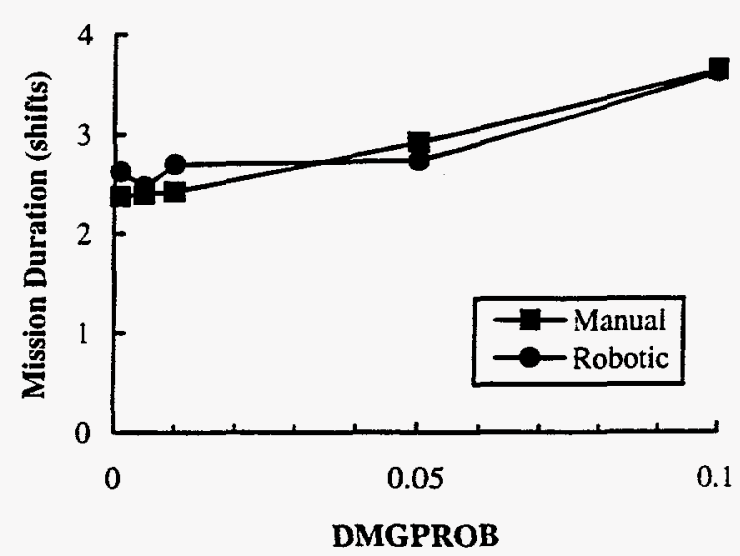

Figure 6. Impact of DMGPROB on mission duration.

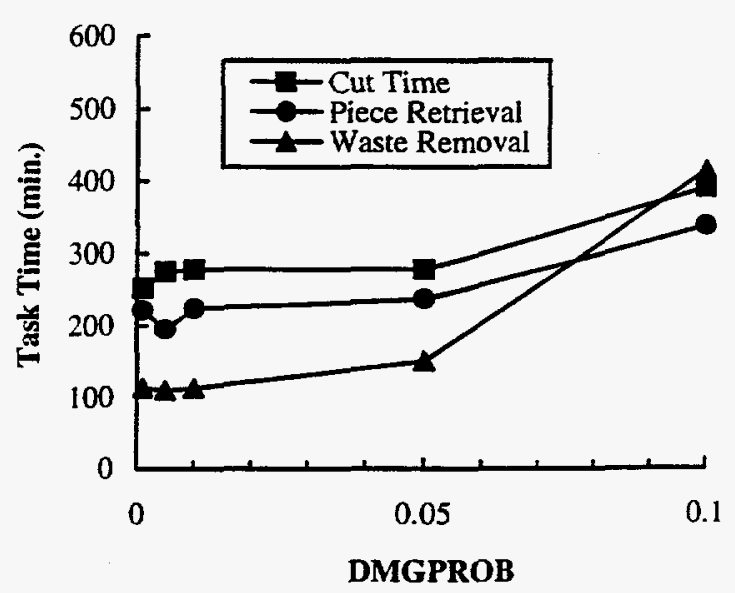

Figure 7. Impact of DMGPROB on task time within supervisory control. 


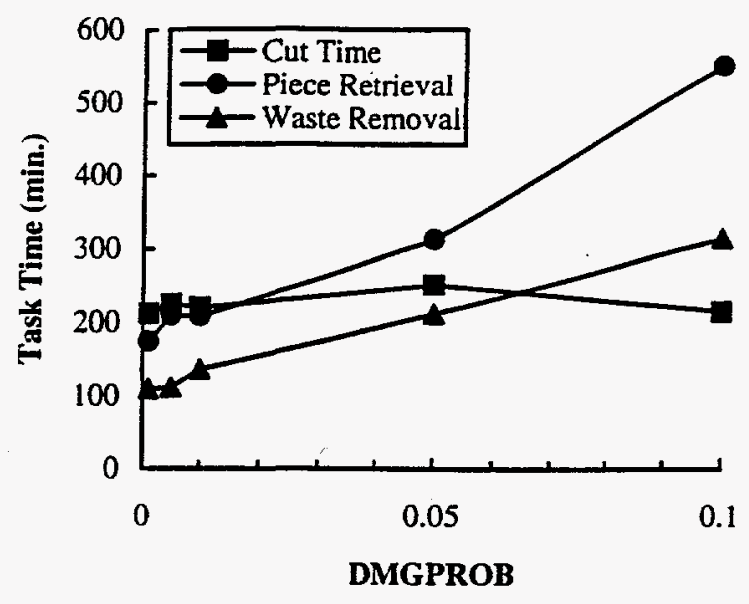

Figure 8. Impact of DMGPROB on task time within teleoperation.

However, these factors were mitigated by two other aspects of the model. First, the mean time required to move the manipulator from a start position to a new position enabling the end-effector to cut/retrieve for supervisory control was $1.54 \mathrm{~min}$ but only $1.10 \mathrm{~min}$ for teleoperation. Teleoperation of the manipulator was faster than supervisory control. Second, teaching sequences of cutting/retrieval/removal operations to the TWRMS were omitted for teleoperation, resulting in a net time savings. These two considerations apparently were enough to offset the timing advantages of supervisory control. There has been speculation in the past that supervisory control might be inefficient unless tasks are repetitive; ${ }^{6}$ the data generated by the model seem to verify that hypothesis.

Although the total time required for waste processing favored teleoperation, user attentional demand may be greater when teleoperation is used. The additional allocation of attentional effort is only manifested when multiple layers of waste are removed under teleoperation. The reason seems to be that continuous control, which is more extensive under teleoperation, was judged by subject matter experts to impose greater attentional demand as compared to monitoring, which characterizes much of user activity during supervisory control. Greater demand could translate into increased requirements for staffing and training, which may offset the speedup advantage of teleoperation.

Increases in robotic position error probability resulted in slowdowns in mission duration, cutting time, and retrieval time, but for a practical range of error rates, the slowdowns were not substantial. Mission duration was severely affected only when the error rate was 0.5 . The percentage of time engaged in repair activity was $11.7 \%$ when RPEP $=0.5$ but was only $14 \%$ for more reasonable values of RPEP.

The results for user position error probability mirrored the findings for RPEP. UPEP caused slowdowns in mission time, cutting time, and piece retrieval time, but the increases were not dramatic except when UPEP $=0.5$, which is probably an unrealistically large user error rate. (Ref. 7 found that the sum of positioning error probabilities during a task requiring many reaches for objects was $p$ $=0.45$ for a dexterous manipulator; this is roughly equivalent to the probability of one error occurring per waste layer.)

Mission duration, cutting time, and retrieval time increased dramatically with damage probability for both supervisory control and teleoperation. Mean mission duration jumped by approximately $50 \%$ when damage probability increased from 0.001 to 0.1 . The relative advantage of teleoperation over supervisory control was masked at the highest level of damage probability, although a damage probability equal to 0.1 exceeds a realistic range for this variable.

\section{Concluding Remarks}

The TWRMS simulation considered two possible control mode cases: supervisory control and teleoperation. It is important to understand that these are not the only options, nor are they mutually exclusive. These cases in fact represent points near the ends of a wide continuum of possible control strategies for the TWRMS. In supervisory control the user is mainly responsible for strategic inputs while in teleoperation the user is responsible for all inputs, including the entire range from low-level commands to set end-effector position to strategic inputs. There are many steps between these two extreme positions. ${ }^{8}$ For example, consider the case of waste retrieval itself. This task may be performed under supervisory control, with the human user indicating what area the system should work in, telling it to begin, and then monitoring operations. In teleoperation, the user could manually control the movements of the end-effector to complete the same task. It is also possible that the user and the computer controller could share some aspects of control. The user could input the direction of travel for the end-effector while the computer maintains separation between waste surface and endeffector and controls the travel rate.

It is likely that the most efficient control method for many TWMRS tasks will be a hybrid operation of the sort described in the preceding example. The task analysis' and 
the task network model described in this paper can provide guidance concerning tasks. When combined with good human factors and engineering judgment, it is possible to use them to identify how manual and robotic control can best be integrated to accomplish the TWMRS mission. The model, combined with performance testing, can also be used to verify the efficiency of control mode options.

The TWRMS simulation is a valuable tool for assessing the impact of components on overall system performance. However, as with all simulations, the quality of its output depends on the quality of its input. The subject matter expert opinions are the best data now available for comparing the relative impact of teleoperation and supervisory control in this context, but they are not the best possible data. For that reason, the mission duration estimates provided by this model should be viewed with caution. With the model in place, it is now possible to collect sub-task data using existing manipulator systems. Feeding these data back into the MicroSAINT model can enhance the performance of the simulation in the future and provide accurate mission duration estimates.

Simplifying assumptions made in the model are another potential source of inaccuracy. However, the model is easily extensible, both in the sense of adding new tasks and branches and increasing the level of detail within existing tasks and branches. Simplified operations in the current model can be turned into new subnetworks in the next model, as better information on the strategies and tactics of waste retrieval becomes available.

These criticisms of the model also illustrate one of the strengths of the model: it points the way for future research by identifying areas where data are insufficient and by identifying critical parts of the task. Future iterative use of the task network model can enhance TWRMS project efficiency by helping to establish future research and development priorities.

\section{ACKNOWLEDGEMENTS}

The Oak Ridge National Laboratory is operated by Martin Marietta Energy Systems, Inc., for the U.S. Department of Energy under subcontract DE-AC0586ER80403. The authors are grateful for the support of $D$.
W. Bennett of Pacific Northwest Laboratories and Dr. B. L. Burks of the ORNL and for the cooperation of the subject matter experts who responded to the task timing survey.

\section{REFERENCES}

1. J. V. DRAPER, Task Analysis for the Single-Shell Tank Waste Retrieval Manipulator System, ORNL/TM12432, Oak Ridge National Laboratory, Oak Ridge, TN, (1994).

2. J. C. SCHRYVER and J. V. DRAPER, Network Simulation Analysis of Level of Control for the SingleShell Tank Waste Retrieval Manipulator System, ORNL/TM-12752, Oak Ridge National Laboratory, Oak Ridge, TN (1994).

3. G. P. CHUBB, K. R. LAUGHERY, and A. A. B. PRITSKER, "Simulating manned systems," in Handbook of Human Factors, G. Salvendy, Ed., John Wiley \& Sons, New York (1987), pp. 1298-1327.

4. K. R LAUGHERY and C. PLOTT, "Task network modeling of human operators in nuclear power plant control rooms," Proceedings of Advances in Human Factors Research on Man/Computer Interactions: Nuclear and Beyond, American Nuclear Society, La Grange Park, IL (1993), pp. 90-95.

5. B. J. WINER, Statistical Principles in Experimental Design. McGraw-Hill, Inc., New York, (1971)

6. T. B. SHERIDAN, Telerobotics, Automation, and Human Supervisory Control, The MIT Press, Cambridge, MA (1992).

7. J. V. DRAPER et al., Final Report: Manipulator Comparative Testing Program, ORNL/TM-10109, Oak Ridge National Laboratory, Oak Ridge, TN (1987).

8. J. V. DRAPER, Teleoperator Hand Controllers: A Contextual Human Factors Assessment, ORNL/TM12762, Oak Ridge National Laboratory, Oak Ridge, TN (1994). 